\title{
Assessment of CT angiographic findings in comparison with echocardiography findings of chest among patients with aortic arch anomalies
}

\author{
Hussein Soleimantabar' ${ }^{1}$, Sofia Sabouri ${ }^{1}$, Leila Khedmat ${ }^{2}$, Samira Salajeghe ${ }^{3}$, Behzad Memari ${ }^{4}$, \\ Bahareh Heshmat Ghahderijani ${ }^{5}$ \\ ${ }^{1}$ Department of Radiology, Emam-Hossein Hospital, Shahid Beheshti University of Medical Sciences, Tehran; ${ }^{2}$ Health \\ Management Research Center, Baqiyatallah University of Medical Sciences, Tehran; ${ }^{3}$ Department of Radiology, Bam \\ University of Medical Sciences, Bam; ${ }^{4}$ Department of Radiology, Zanjan University of Medical Science, Zanjan; \\ ${ }^{5}$ Department of Radiology, Imam Ali Hospital, Zahedan University of Medical Sciences, Zahedan, Iran
}

\begin{abstract}
The objective of this study was to examine the findings of computed tomographic (CT) angiography in patients with aortic arch anomalies in comparison with transthoracic echocardiography findings who referred to a private imaging center in Tehran during 2009-2012. The cases included 203 patients with clinical symptoms or echocardiogram of congenital heart disease to assess the presence of aortic arch anomalies among patients referred to imaging center. This study is a retrospective study based on the CT angiographic findings in comparison with transthoracic echocardiography findings of chest among patients with aortic arch anomalies. In this study, 203 patients with congenital anomalies were enrolled in the study, among those, 107 patients were men and 96 were female. The most common anomaly of the aortic arch was found to be coarctation $(19.7 \%)$, followed by right sided arch with mirror image branching (19.2\%). Furthermore, the most common cardiac anomalies associated with aortic arch anomalies were VSD, PA and PDA.
\end{abstract}

Correspondence: Bahareh Heshmat Ghahderijani, Department of Radiology, Imam Ali Hospital, Zahedan University of Medical Sciences, Zahedan, Iran.

E-mail: bahar.heshmatmd@gmail.com

Contributions: All authors contributed equally to research design and method, data analysis and manuscript drafting. All authors read and approved the final version to be published.

Conflict of interest: The authors declare no conflict of interest.

Keywords: Echocardiography; CT angiography; coronary artery; thoracic vessels.

Received for publication: 25 June 2019.

Accepted for publication: 5 September 2019

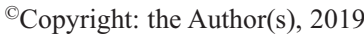

Licensee PAGEPress, Italy

Monaldi Archives for Chest Disease 2019; 89:1120

doi: 10.4081/monaldi.2019.1120

This article is distributed under the terms of the Creative Commons Attribution Noncommercial License (by-nc 4.0) which permits any noncommercial use, distribution, and reproduction in any medium, provided the original author(s) and source are credited.
The sensitivity and specificity of transthoracic echocardiography in the diagnosis of aortic arch anomalies was 59\% and 100\% in comparison with $\mathrm{CT}$ angiography. In addition, the agreement between the two methods (kappa) in the diagnosis of aortic arch anomalies was 0.72 . But, transthoracic echocardiography is the first diagnostic method for patients with congenital heart disease. In some patients, the ability of this method was limited to the detection of coronary artery anomalies and thoracic vessels. Therefore, CT is used for morphological evaluation of congenital heart disease (CHD) due to its main advantages including fast acquisition time, large anatomical coverage, high speed, and great spatial resolution. Moreover, CT is essential for proper evaluation of CHD regarding its high spatial and temporal resolution.

\section{Introduction}

Congenital heart disease (CHDs) still imposes significant cardiac problems in the children's age group. Transthoracic echocardiography has been used to diagnose and follow up patients as primary non-invasive imaging devices [1]. CHD are commonly seen in $1 \%$ of all live births, including acyanotic and cyanotic congenital birth defects, depending on the presence or absence of cyanosis in the physical assessment $[2,3]$.

Transthoracic echocardiography is considered as the primary imaging method for the evaluation and diagnosis of CHD, allowing anatomic and functional evaluation. This operator-dependent imaging method is restricted by an acoustic window with the benefits of being able to provide noninvasive and portable technique [3-5], and to be capable of providing high-resolution anatomical and physiological data [4]. Nevertheless, this method has some disadvantages as its definitions for the great arteries and intra cardiac anomalies, pulmonary and coronary arteries are not considered to be accurate $[6,7]$.

In the past, angiography has been the main modality for diagnosing CHD, and aortic disorders, but is now less likely to be used due to some limitations including its requirement for general anesthesia, invasiveness, higher exposure of neonates to radiation and iodinated contrast materials $[3,8]$. With technological advancements, computed tomographic (CT) and MRI have taken into consideration in this regard. In particular, CT is widely used in the diagnosis of thoracic aortic diseases, especially its newer generations, including spiral CT angiography and Multi-detector computed tomography (MDCT) $[9,10]$. Performing a simple CT scan can be simply done for most patients, but for more information, 
CT is more helpful with the contrast agent. Additionally, dynamic $\mathrm{CT}$ provides an opportunity for further evaluation of the blood flow in the cardiovascular system [9]. MDCT is the current imaging modality used to detect many cases in the thoracic aorta including aortic aneurysm, aortic dissection, aortic intramural hematoma, atherosclerotic plaques, Traumatic aortic injury (TAI) and congenital malformations [11-13]. MDCT angiography has been developed to evaluate thoracic aortic abnormalities. This non-invasive modality is capable of assessing vascular anomalies and the tracheal and/or esophageal compression [14-16]. The main advantages added by MDCT to diagnosis have been attributed to high levels of anatomical coverage, high speed, great spatial resolution, and its multi-planar reformatting as well as its 3D volume rendering to provide opportunity for solving pitfall associated with poor temporal resolution in CT [7,17].

The great disadvantages of MDCT have been characterized by contrast material requirement, absence of hemodynamic data and ionizing radiation exposure, particularly in pediatrics [17-19]. MDCT angiography has been widely used in evaluating thoracic aortic anomalies, where in was capable of replacing cardiac catheterization in assessment of aortic diseases anomalies [14,20,21].

However, MDCT angiography need iodized contrast and higher radiation exposure that can be considered as disadvantages of this method [16]. The temporal resolution of cardiac CT angiography has been defined to be less than echocardiography, but it has been still considered as a combination of advantages such as fast acquisition time, large anatomical coverage, high speed, great spatial resolution, and 3D ability added to flexible ECG synchronization, leading to increased quality of imaging and decreasing the risk of subjects [20].

Therefore, the aim of our study was to assess the role of CT angiography to patients with aortic arch anomalies in comparison with echocardiography findings in subjects referred to a private imaging center in Tehran, Iran.

\section{Materials and Methods}

Ethics approval was obtained from the institutional review board of Shahid Beheshti University of Medical Sciences according to Helsinki declaration. Written informed consent was signed by the parents at study entry.

In this retrospective study, 203 patients referred to Tooska Imaging Center during 2009 and 2012 with clinical symptoms or echocardiographic evidence of congenital heart disease was enrolled to evaluate the presence of aortic arch anomalies. Furthermore, CT angiography findings were evaluated in combination with chest transthoracic echocardiography of patients with aortic arch anomalies.

In this study, the characteristics of individuals were recorded based on a questionnaire according to the variables such as age, sex, etc., and after examination, CT angiography findings were also recorded by an experienced radiologist at the cardiac CT angiography. Inclusion criteria included patients with clinical signs or transthoracic echocardiography suggesting congenital heart disease. Exclusion criteria was subjects having sensitivity to contrast agents and poor renal function. Patients included in our study consisted of 107 males and 96 females with age ranging between 6-day and 10years-old.

To obtain CT images in all patients, the Light Speed QXI fourrow detector was used. The protocol for performing scans was as follows: in children under 7 years of age, anesthesia was performed, in those over 7 years of age CT scan was performed without anesthesia. Visipaque $(2-2.5 \mathrm{cc} / \mathrm{kg})$ as contrast agent was injected by Power
Injector. The injection speed of the contrast material was proportional to the length of the scan, between 1-3 cc/s.

The CT scan sections included the middle line of the neck to the iliac crest. The maximum intensity projection (MIP), multiplanar reconstruction (MPR), and volume rendering (VR) images were created from raw sections in the workstation 4.1. Scan conditions include: I, Collimation (1.25 mm with $50 \%$ overlapping); ii, Table speed $(3.75 \mathrm{~cm} / \mathrm{s})$; Pitch $(0.75)$.

\section{Statistical analysis}

Qualitative data were presented in the study as relative frequencies (percentage). Statistical analysis conducted by using SPSS 18.0 for windows (SPSS Inc., Chicago, IL, USA).

\section{Results}

In this study, 203 patients with congenital heart anomalies were included. Of these, 107 (52.7\%) were male and 96 (47.3\%) were female. The median age of the participants in the study was 24 months. Table 1 and Figure 1 show the congenital anomalies of the aortic arch by CT angiography and transthoracic echocardiography (TEC). The most common anomalies were coarctation of aorta (COA) and right-sided aortic arch with mirror image branching.

In some patients, two or more simultaneous anomalies were diagnosed as following: Anomalies of coarctation along with ventricular arrhythmias (VA) anomaly (4 cases), coarctation with tubular hypoplasia (9 patients), coarctation with aberrant right subclavian artery (ARSA), (4 patients), coarctation with hypoplastic arch, coarctation with cervical arch (4 subjects), VA anomaly with ARSA (1 cases). VA anomaly with hypoplastic arch (1 subjects), VA anomaly with right-sided aortic arch with mirror image branching (3 patients), VA with cervical arch (3 cases), tubular hypoplasia with ARSA (2 patients), tubular hypoplasia with a cervical arch (1 case), aberrant right subclavian artery (ARSA) with cervical arch (2 patients), ARSA with right SCA (2 patients), ARSA with interruption (3 cases), hypoplastic arch with interruption (1 cases), right-sided aortic arch with mirror image branching with truncus arteriosus (1 patient), and right sided left SCA with truncus arteriosus (1 case).

Among the enrolled patients, 69 (34\%) were normal with CT scan imaging, while $66(32.5 \%)$ were normal by transthoracic echocardiography imaging. Among patients with VA originating from arch, $13(6.6 \%)$ showed left VA originating from arch using CT angiography, followed by right VA originating from arch right sided (3 subjects, 1.5\%). By using the transthoracic echocardiography, this anomaly was not detected in any patients.

Among patients with interruption, 3 (1.5\%) were found as type A, $2(1 \%)$ were type B and 3 were type C $(1.5 \%)$ based on the CT angiography, while echocardiographic imaging showed that one patient had type B, and type $\mathrm{C}$ each. Among types B and C, 2 cases of interruption were detected, but their type was not determined.

In the comparison of diagnostic value for congenital heart anomalies of CT angiography and transthoracic echocardiography, we found that 164 patients were negative with CT angiography and TEC and 28 patients were found positive with CT angiography compared with transthoracic echocardiography. In addition, 2 patients exhibited anomalies with CT angiography and TEC and 9 patients were positive with CT angiography and TEC.

The most common associated cardiac anomalies among 
patients with aortic arch anomalies were pulmonary atresia (PA), ventricular septal defect (VSD) and patent ductus arteriosus (PDA) anomalies (Table 2 and Figure 2).

Sensitivity and specificity of transthoracic echocardiography compared with $\mathrm{CT}$ angiography based on aortic arch anomalies: the sensitivity and specificity of transthoracic echocardiography were $59 \%$ and $100 \%$, respectively, in the detection of aortic arch anomalies. The agreement between two methods (Kappa coefficient) was

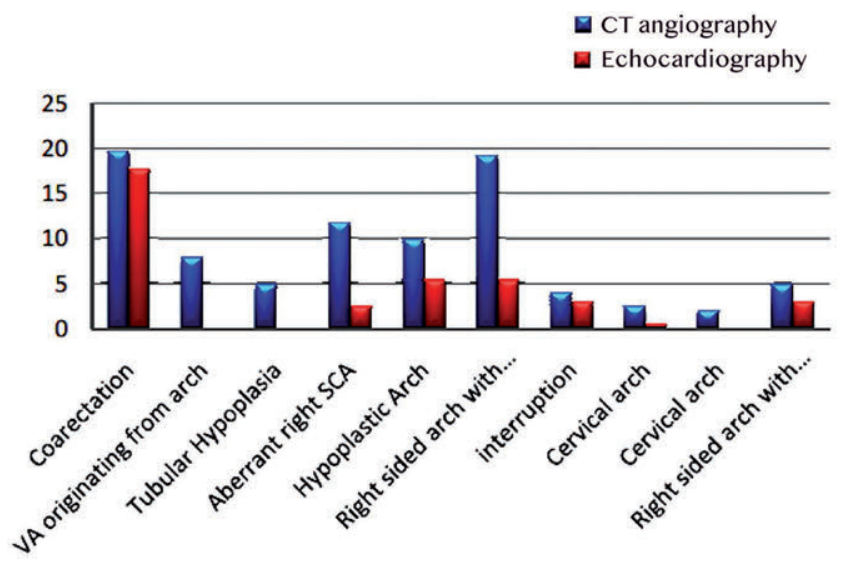

Figure 1. The prevalence of aortic arch anomalies using CT angiography and echocardiography. VA, ventricular arrhythmias; CT, computed tomographic; SCA, left subclavian artery.

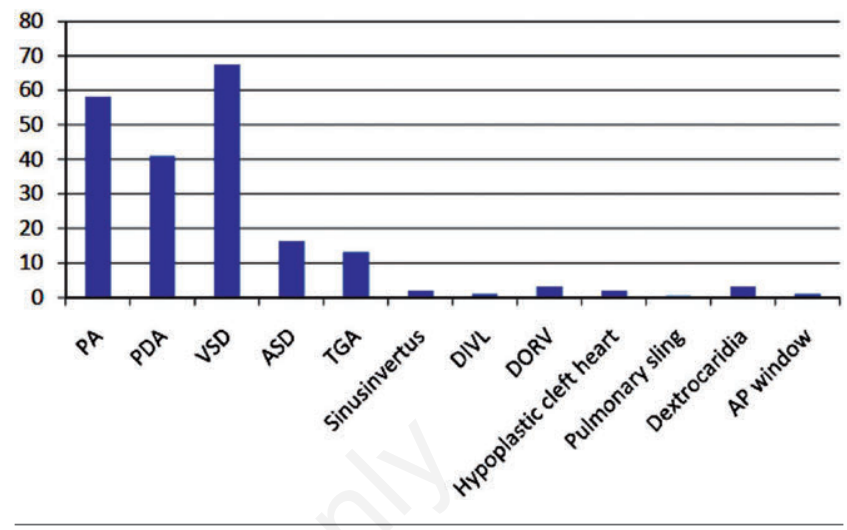

Figure 2. Status of associated cardiac abnormalities in patients with aortic arch anomalies. PA, pulmonary atresia; VSD, ventricular septal defect; PDA, patent ductus arteriosus; ASD, atrial septal defect; TGA, transposition of the great arteries; DILV, double inlet left ventricle; DORV, double outlet right ventricle; AP, aortopulmonary.

Table 1. Congenital anomalies of the aortic arch by CT angiography and echocardiography.

\begin{tabular}{lcccc} 
Anomaly of the aortic arch & \multicolumn{2}{c}{ CT angiography } & \multicolumn{2}{c}{$\begin{array}{c}\text { Echocardiography } \\
\text { Yes }\end{array}$} \\
Coarctation & Yes & No & $36(17.7 \%)$ & $167(82.3 \%)$ \\
VA originating from arch & $40(19.7 \%)$ & $163(80.3 \%)$ & $0 \%$ & $203(100 \%)$ \\
\hline Tubular hypoplasia & $16(7.9 \%)$ & $187(91.1 \%)$ & $0 \%$ & $203(100 \%)$ \\
Aberrant right SCA & $10(4.9 \%)$ & $193(95 \%)$ & $5(2.5 \%)$ & $197(97.5 \%)$ \\
\hline Hypoplastic arch & $24(11.8 \%)$ & $179(88.2 \%)$ & $11(5.4 \%)$ & $192(94.6 \%)$ \\
Right sided arch with mirror image branching & $20(9.9 \%)$ & $183(90.1 \%)$ & $11(5.4 \%)$ & $192(94.6 \%)$ \\
\hline Interruption & $39(19.2 \%)$ & $164(80.8 \%)$ & $6(3 \%)$ & $197(97 \%)$ \\
Cervical arch & $8(4 \%)$ & $195(96 \%)$ & $1(0.5 \%)$ & $202(99.5 \%)$ \\
\hline Bovine arch & $5(2.5 \%)$ & $198(97.5 \%)$ & $0 \%$ & $203(100 \%)$ \\
Right sided arch with aberrant left SCA & $2(1 \%)$ & $201(99 \%)$ & $6(3 \%)$ & $197(97 \%)$ \\
\hline Double aortic arch & $10(4.9 \%)$ & $193(95.1 \%)$ & $0 \%$ & $203(100 \%)$ \\
\hline
\end{tabular}

CT, computed tomographic; VA, ventricular arrhythmia; SCA, left subclavian artery.

Table 2. Status of cardiac associated anomalies in patients with aortic arch anomalies.

\begin{tabular}{|c|c|c|c|c|c|c|}
\hline Associated cardiac anomalies & PA & PDA & VSD & ASD & TGA & Situs inversus \\
\hline Yes & $118(58.1 \%)$ & $83(40.9 \%)$ & $137(67.5 \%)$ & $33(16.3 \%)$ & $27(13.3 \%)$ & $4(2 \%)$ \\
\hline No & $85(41.9 \%)$ & $20(59.1 \%)$ & $66(32.5 \%)$ & $170(83.7 \%)$ & $176(86.7 \%)$ & $199(98 \%)$ \\
\hline Associated cardiac anomalies & DILV & DORV & $\begin{array}{l}\text { Hypoplastic } \\
\text { left heart }\end{array}$ & $\begin{array}{l}\text { Pulmonary } \\
\text { sling }\end{array}$ & Dextrocardia & AP window \\
\hline Yes & $2(1 \%)$ & $7(3.4 \%)$ & $4(2 \%)$ & $1(0.5 \%)$ & $7(3.4 \%)$ & $2(1 \%)$ \\
\hline No & $201(99 \%)$ & $196(96 \%)$ & $199(98 \%)$ & $202(99.5 \%)$ & $196(96.6 \%)$ & $201(99 \%)$ \\
\hline
\end{tabular}

$\mathrm{PA}$, pulmonary atresia; VSD, ventricular septal defect; PDA, patent ductus arteriosus; $\mathrm{ASD}$, atrial septal defect; TGA, transposition of the great arteries; DILV, double inlet left ventricle; DORV, double outlet right ventricle; AP, aortopulmonary. 
0.72 for the diagnosis of aortic arch anomalies. Sensitivity and specificity of transthoracic echocardiography were calculated as $90 \%$ and $100 \%$ for coarctation detection. We found an agreement between the two methods (Kappa coefficient: $0.93 \%$ ).

The specificity of transthoracic echocardiography for the diagnosis of tubular hypoplasia was determined as $100 \%$. The specificity of transthoracic echocardiography for VA originating from arch anomaly was calculated as $100 \%$, but its sensitivity was very low. In fact, 16 patients with this anomaly were diagnosed by CT angiography, while they were negative by echocardiography. The sensitivity and specificity of transthoracic echocardiography for the detection of hypoplastic arch anomalies were $55 \%$ and $100 \%$. The agreement between the two methods for detecting this anomaly was 0.68 (Kappa coefficient). The sensitivity of transthoracic echocardiography for the detection of right-sided aortic arch with mirror image branching was $28.2 \%$ and its specificity was $100 \%$. The agreement between the two methods in detecting this anomaly was 0.38 (Kappa coefficient). Sensitivity and specificity of transthoracic echocardiography were $75 \%$ and $100 \%$ for interruption, respectively. Sensitivity and specificity of the echocardiogram for the diagnosis of cervical arch anomaly were calculated to be $20 \%$ and $100 \%$. We found an agreement between both methods at level of 0.32 (Kappa coefficient). Specificity of echocardiography for anomalies Bovine arch was determined as $100 \%$, but its sensitivity was not ascertainable, because 2 patients with this anomaly were diagnosed using CT angiography, while echocardiography was not capable of detecting these patients (Table 3). Sensitivity and specificity of transthoracic echocardiography were $60 \%$ and $100 \%$, respectively, for the detection of right sided arch with aberrant left SCA. The agreement between the two methods in detecting this anomaly was found to be 0.74 (Kappa coefficient). Sensitivity and specificity of transthoracic echocardiography for the detection of truncus arteriosus (TA) were $100 \%$ and $100 \%$, respectively. The agreement between the two methods was recorded to be 1 (Kappa coefficient). Aortic arch anomalies in different age groups: the age of the subjects was classified into 3 groups, under 6 months, 7 to 24 months and more than 24 months.

\section{Discussion}

In the present study, we report the role of CT angiography in comparison with transthoracic echocardiography in patients with aortic arch anomalies referred to a private imaging center in Tehran, Iran.

The most common aortic arch anomalies and the most commonly associated cardiac anomalies were assessed. The most common anomalies of aortic arch were found to be coarctation (19.7\%), followed by right sided arch with mirror image branching (19.2\%). Furthermore, the most common associated cardiac anomalies among patients with aortic arch anomalies were PA, VSD and PDA. Based on the data presented in the study, sensitivity and specificity of transthoracic echocardiography findings were calculated. Coarctation of the aorta is a congenital heart disease implicating a narrowing of the aorta, which is often shown an isolated defect or in association whit different other lesions. Early diagnosis, information about the associated anomalies and the severity of the disease are of great importance for providing therapeutic strategies. In this regard, several non-invasive methods, such as transthoracic echocardiography, CT and MRI are widely used. Transthoracic echocardiography as an accessible and safe method is commonly applied as a screening technique. This method is also useful for intraoperative and hemodynamic studies, but CT is highly recommended prior to any surgical or therapeutic approach. In a study by Darabian et al., 2013 has been reported that cardiac CT angiography revealed an excellent spatial resolution and a good power for finding associated anomalies [22].

In our study, coarctation represents the most common aortic arch anomaly accounting for $19.7 \%$ of our cases. Abbruzzese and Aidala [23] indicated that the prevalence of aortic coarctation among congenital heart disease was $6.5 \%$. Aortic coarctation is considered to be the most frequently observed congenital defect, accounting for approximately $7 \%$ of all congenital cardiac lesions. This anomaly consists of a focal stenosis that is mostly presented at the aortic isthmus, nonetheless, can also be seen in tubular fashion [23]. Intravascular stent therapy is recommended for patients with coarctation of the aorta [24,25].

Mirror-image branching of the arch vessels is considered to be the most frequent kind of right aortic arch, which is associated with CHD in nearly $95 \%$ of cases. As seen in our study, most of the patients with the right aortic arch pattern are in childhood. Based on the previous findings, around $25 \%$ of individuals with tetralogy of Fallot and $25-50 \%$ of subjects with truncus arteriosus exhibit a right aortic arch along with mirror-image branching [26]. In our study, the most common associated cardiac anomalies were PA (98.7\%), followed by VSD (87.2\%), and PDA (28.2\%).

Table 3. Aortic arch anomalies in different age groups.

\begin{tabular}{lccc} 
Anomaly of the aortic arch & Less than $\mathbf{6}$ months & From 7 to 24 months & Over 24 months \\
Coarctation & $23.1 \%$ & $23 \%$ & $53.9 \%$ \\
VA originating from arch & $31.3 \%$ & $25 \%$ & $43.9 \%$ \\
\hline Tubular hypoplasia & $40 \%$ & $40 \%$ & $20 \%$ \\
Aberrant right SCA & $41.7 \%$ & $33.3 \%$ & $25 \%$ \\
\hline Hypoplastic arch & $42.1 \%$ & $26.4 \%$ & $31.6 \%$ \\
Right sided arch with mirror image branching & $17.9 \%$ & $30.8 \%$ & $51.3 \%$ \\
\hline Interruption & $50 \%$ & $25 \%$ & $25 \%$ \\
Cervical arch & $0 \%$ & $20 \%$ & $80 \%$ \\
\hline Bovine arch & $0 \%$ & $100 \%$ & $0 \%$ \\
Right sided arch with aberrant left SCA & $10 \%$ & $40 \%$ & $50 \%$ \\
\hline
\end{tabular}

VA, ventricular arrhythmia; SCA, left subclavian artery. 
The utility of 16-slice MDCT angiography to characterize the anatomic properties of aberrant subclavian arteries has been described in 17 patients [16]. In this study 11 subjects showed aberrant right subclavian artery from the left aortic arch, 6 an aberrant left subclavian artery from the right aortic arch (6 subjects), Kommerell's diverticulum in 3 subjects with an aberrant right subclavian artery and in 5 subjects with an aberrant left subclavian artery, esophageal compression in 8 cases, aneurysm in 2 patients and tracheal compression in one child, [16]. In addition, aberrant subclavian artery was correlated with complex CHD (1 subjects), intracardiac defects in two subjects, patent ductus arteriosus in 2 subjects, coarctation in 2 subjects, and aberrant vertebral artery in 1 case.

In our study, 4 patients had both coarctation and aberrant right subclavian artery. The aberrant left subclavian artery results from the interruption between the left carotid artery and the left subclavian artery in creating a double aortic arc. The left-sided aortic arch in combination with right subclavian artery is the most common aortic arch anomaly, accounting for an incidence of 0.5 to $2 \%$. Usually the right subclavian artery is separately created, but sometimes accompanied by other cardiovascular anomalies [16]. Aberrant subclavian artery usually accompanies aortic coarctation ( 4 cases in our study), patent ductus arteriosus ( 10 cases in our study), and intracardiac defect. In our study, the most common cardiac anomalies with aberrant right subclavian artery were PA (54.2\%), PDA (41.7\%) and VSD (62.5\%). In addition, based on the data presented herein, an aberrant right subclavian artery with tubular hypoplasia was found in one patient, an aberrant right SCA with cervical arch in 2 patients and aberrant right subclavian arteries with interruption in 3 cases.

MDCT angiography is considered as a valid and non-invasive technique due to its advantages such as fast acquisition time, great spatial resolution and wide availability. Therefore, it is the main imaging method to evaluate vascular and thoracic anomalies. Therefore, the identification of the aberrant right subclavian arteries is of clinical significance because it is are associated with tracheoesophageal compression, aneurysm, ruptured aneurysm, and other anomalies. CT angiography is able to illustrate the anatomical details of the vascular structures, and the relationship between them and adjacent organs. This is the most important advantage of CT angiography in comparison with other imaging modalities in the evaluation of the right subclavian artery [14-16]. Right-sided arch with aberrant left SCA is the most common right-sided aortic arch in adults, with left SCA originating from the last vessel of the arch and crossing the mediastinum from right to left. The incidence of congenital heart anomalies is considered to be low, as our findings revealed, with most patients showing tetralogy of Fallot. Sometimes the origin of the aberrant left subclavian arteries is the diverticulum of Kommerel; most patients with this anomaly are usually asymptomatic, indicating that the vascular ring is completely loose [26]. In our study, the accompanying anomalies are PA and VSD.

Hypoplasia of the aortic arch is associated with other types of obstruction or aortic flow limitation, mainly coarctation. Other congenital heart anomalies, such as ASD, VSD and PDA, are associated with this anomaly [21]. In our study, tubular hypoplasia with PDA was seen in 6 cases. Double aortic arch is the most common type of vascular ring and is not usually accompanied with other heart anomalies $[9,27,28]$. In our study, we had a case with double aortic arch that did not show any other cardiac anomalies.

Interrupted aortic arch (IAA) is a type of aortic coarctation characterized by the discontinuity of the aortic arch between the ascending and descending aorta. The IAA is classified based on the location of the interruption, being type $\mathrm{A}$ in around $42 \%$ of infants, type B in $53 \%$ of patients (most common type), type C, the least common type, in $5 \%[29,30]$. In our study, 3 cases were found as type A (37.5\%), followed by type B (2 cases; $25 \%)$ and type C (3 cases; $37.5 \%$ ). Additionally, the most common cardiovascular anomalies included PDA and VSD, which was in agreement with Kimura-Hayama et al. [21].

MDCT angiography is widely used to diagnose aortic interruption. Three-dimensional measurements of MDCT well show the origin of large arteries and collateral vessels. MDCT angiography may be used as post-surgical follow-up examination or preoperative care [30]. Our findings demonstrated that the sensitivity and specificity of transthoracic echocardiography for the detection of interruption were $75 \%$ and $100 \%$, in comparison CT angiography.

Truncus arteriosus is considered a rare form of congenital heart defect, accounting for $1.7-4.6 \%$ of all CHD. Furthermore, $12 \%$ of patients with TA show a number of anomalies such as associated underdevelopment of the aortic arch as in IAA, severe hypoplasia of arch, aortic atresia, and/or coarctation, often associated to a PDA [31]. In our study, two patients with TA were found, one of them with PA and VSD. One of these 2 cases showed a right-sided arch with mirror image branching with TA, and the other a rightsided left SCA. Regarding our findings, the sensitivity and specificity of transthoracic echocardiography was $100 \%$ and $100 \%$. Diagnostic sensitivity (27.8\%), specificity (97.6\%) and accuracy of Doppler-echocardiography $(76.7 \%)$ were determined as reported by Yang et al. [32].

The aortic arch anomalies are sometimes very complex and may be associated to venous and to tracheal abnormalities. In these conditions, the determination of morphology and association of aortic arch abnormalities can be very difficult by applied two-dimensional angiographic images or cross-sectional imaging of CT or MRI. CT angiography is the most useful imaging modality capable of demonstrating the morphological anatomy of vascular structures and their relationship with adjacent organs, and of showing normal and pathologic morphologic characteristics of cardiovascular structures [33]. This ability is the greatest advantage of CT angiography in evaluating congenital anomalies. In addition, 3D images are considered to be very useful for surgical decision making.

Transthoracic echocardiography is the first diagnostic method for patients with congenital heart disease, but in some patients the ability of this method can be limited for detecting anomalies of the aortic arch and thoracic vessels. It may also be used to evaluate descending aorta, abnormal vascular anatomy and aortic coarctation. MRI, despite anatomical coverage and the capability of functional evaluation, has limited applicability in patients who do not cooperate. MRI studies are time-consuming and require patient relief [34-37]. The bolus tracking CT technique, on the other hand, has enhanced the role of MDCT for coarctation detection [15]. In our study, the sensitivity and specificity of the transthoracic echocardiography for coarctation detection was $90 \%$ and $100 \%$, respectively. The agreement of two methods in quark detection was 0.93 (Kappa coefficient). This finding suggests that transthoracic echocardiography can be successful as CT scan angiography in diagnosing aortic coarctation.

In conclusion, transthoracic echocardiography missed the diagnosis of some cases, with particular limitations in the detection of coronary artery anomalies and thoracic vessels. Therefore, CT has many advantages over echocardiogram, such as fast acquisition time, large anatomical coverage, high speed, great spatial resolution, resulting in higher quality of imaging.

Based on our findings, the greatest value of $\mathrm{CT}$ angiography lies in the detection of aortic arch anomalies. Using CT angiogra- 
phy together with transthoracic echocardiography can be useful in improving the diagnostic accuracy.

\section{References}

1. Khatri S, Varma SK, Khatri P, et al. 64-slice multidetector-row computed tomographic angiography for evaluating congenital heart disease. Pediatr Cardiol 2008;29:755-62.

2. Madsen NL, Marino BS, Woo JG, et al. Congenital heart disease with and without cyanotic potential and the long-term risk of diabetes mellitus: A population-based follow-up study. J Am Heart Assoc 2016;5. pii:e003076.

3. Enaba MM, Hasan DI, Alsowey AM, et al. Multidetector computed tomography (CT) in evaluation of congenital cyanotic heart diseases. Pol J Radiol 2017;82:645-59.

4. Chandrashekhar G, Sodhi KS, Saxena AK, et al. Correlation of 64 row MDCT, echocardiography and cardiac catheterization angiography in assessment of pulmonary arterial anatomy in children with cyanotic congenital heart disease. Eur J Radiol 2012;81:4211-7.

5. Park MK. The pediatric cardiology handbook. 4th ed. Philadelphia: Mosby Elsevier; 2010.

6. Wiant A, Nyberg E, Robert G. CT evaluation of congenital heart disease in adults. Am J Roentgenol 2009;193:388-96.

7. Hassanien OA, El-Shafey KHL, Khedr RA, et al. Role of 320MDCT in assessment of cardiac great arteries anomalies. Egyptian J Radiol Nuclear Med 2018;49:993-1002.

8. Rao PS. Diagnosis and management of cyanotic congenital heart disease: Part II. Indian J Pediatrics 2009;76:297-308.

9. Haaga JR, Dogra VS, Forsting M, et al. CT and MRI of whole body. 5th ed. St Louis: Mosby, Elsevier; 2009.

10. Cinar A, Haliloglu M, Karagoz T, et al. Interrupted aortic arch in a neonate: multidetector CT diagnosis. Pediatr Radiol 2004;34:901-3.

11. Abbara S, Kalva S, Cury RC, et al. Thoracic aortic disease: spectrum of multidetector computed tomography imaging findings. J Cardiovasc Comput Tomogr 2007;1:40-54.

12. Valente T, Rossi G, Lassandro F, et al. MDCT evaluation of acute aortic syndrome (AAS). Br J Radiol 2016; 89:2015-25.

13. Chung JH, Ghoshhajra BB, Rojas CA, et al. CT angiography of the thoracic aorta. Radiol Clin North Am 2010;48: 249-64.

14. Lee EY, Siegel MJ, Hildebolt CF, et al. MDCT evaluation of thoracic aortic anomalies in pediatric patients and young adults: comparison of axial, multiplanar, and 3D images. Am J Roentgenol 2004;182:777-84.

15. Gilkeson RC, Ciancibello L, Zahka K. Multidetector CT evaluation of congenital heart disease in pediatric and adult patients. Am J Roentgenol 2003;180:973-80.

16. Türkvatan A, Büyükbayraktar FG, Olçer T, et al. Congenital anomalies of the aortic arch: evaluation with the use of multidetector computed tomography. Korean J Radiol 2009;10:176-84.

17. Goitein O, Salem Y, Jacobson J, et al. The role of cardiac computed tomography in infants with congenital heart disease. Isr Med Assoc J 2014;16:147-52.

18. Brenner DJ, Hall EJ. Computed tomography: an increasing source of radiation exposure. N Engl J Med 2007;357:2277-84.

19. Hausleiter J, Meyer T, Hermann F, et al. Estimated radiation dose associated with cardiac CT angiography. JAMA 2009;30:500-7.

20. Shehata SM, Zaiton FM, Warda MHA. Value of MDCT as a non- invasive modality in evaluation of pediatric congenital cardiovascular anomalies. Egypt J Radiol Nucl Med 2017;48:467-78.

21. Kimura-Hayama ET, Meléndez G, Mendizábal AL, et al. Uncommon congenital and acquired aortic diseases: role of multidetector CT angiography. Radiographics 2010;30:79-98.

22. Darabian S, Zeb I, Rezaeian P, et al. Use of noninvasive imaging in the evaluation of coarctation of aorta. J Comput Assist Tomogr 2013;37:75-8.

23. Abbruzzese PA, Aidala E. Aortic coarctation: an overview. J Cardiovasc Med (Hagerstown) 2007;8:123-8.

24. Gatzoulis M, Swan L, Therrien J, et al. Adult congenital heart disease: a practical guide. Oxford: Blackwell Publishing; 2005.

25. Fukui T. Management of acute aortic dissection and thoracic aortic rupture. J Intensive Care 2018;6:15.

26. Kanne JP, Godwin JD. Right aortic arch and its variants. J Cardiovasc Comput Tomogr 2010;4:293-300.

27. Backer CL, Mongé MC, Popescu AR. Vascular rings. Semin Pediatr Surg 2016;25:165-75.

28. Savla JJ, Weinberg PM. Editorial on "vascular ring diagnosis and management: notable trends over 25 years". Transl Pediatr 2017;6:83-85.

29. Jaffe RB, Condon VR. The heart and great vessels. In: Kuhn JP, Slovis TL, Haller JO, editors. Caffey's pediatric diagnostic imaging. USA: Elsevier Inc. Mosby, 2004; pp. 1284-359.

30. Oguz B, Haliloglu M, Karcaaltincaba M. Paediatric multidetector CT angiography: spectrum of congenital thoracic vascular anomalies. Br J Radiol 2007;80:376-83.

31. Murugan MK, Gulati GS, Ramakrishnan S. Multidetector computed tomography for truncus arteriosus and associated complex arch anomaly. Pediatr Cardiol 2013;34:764-6.

32. Yang M, Mo XM, Jin JY, et al. [Diagnostic value of 64 multislice CT in typing of congenital aortic anomaly in neonates and infants].[Article in Chinese]. Zhonghua Yi Xue Za Zhi 2010;90:2167-71.

33. Shirani S, Soleymanzadeh M. Diagnosis of aortic interruption by CT angiography. Pol J Radiol. 2013;78:72-4.

34. Mehrnahad M, Soleimantabar H, Sanei Taheri M, Ghahderijani $\mathrm{BH}$. Pre-labor rupture of uterus at 32 weeks with extrusion of fetus with intact amniotic sac: a case report. J Inter Trans Med 2019;7:99-102.

35. Mehrnahad M, Soleimantabar H, Ebrahimi A, Ghahderijani BH. Circumferential meningioma of the cervical spinal cord with widespread intracranial extension. J Res Med Dent Sci 2019;7:44-46.

36. Jamaati HR, Heshmat B, Tamadon R, et al. Association between severity of chronic obstructive pulmonary disease and lung function tests. Tanaffos 2013;12:36-41.

37. Moghtadaei M, Otoukesh B, Bodduhi B, et al. Evaluation of patellar position before and after medial opening wedge high tibial osteotomy: Radiographic and computed tomography findings. Med Arch 2016;70:293-5. 